DIGITALCOMMONS

— @WAYNESTATE —
Michigan Journal of Counseling:

Research, Theory and Practice

$2-1-2002$

\title{
Differences among African American Junior High School Students: The Effects of Skin Tone on Ethnic Identity, Self-Esteem and Cross-Cultural Behavior
}

\author{
Alfiee M. Breland \\ Michigan State University, breland@msu.edu \\ Hardin L.K. Coleman \\ University of Wisconsin-Madison \\ Stephanie Irby Coard \\ New York University Medical Center \\ Robbie J. Steward \\ Michigan State University
}

Follow this and additional works at: https://digitalcommons.wayne.edu/mijoc

\section{Recommended Citation}

Breland, A. M., Coleman, H. L. K., Coard, S. I., \& Steward, R. J. (2002). Differences among African American Junior High School Students: The Effects of Skin Tone on Ethnic Identity, Self-Esteem and Cross-Cultural Behavior, Dimensions of Counseling, 30(1), 15-21. doi: $10.22237 / \mathrm{mijoc} / 1012521780$ 


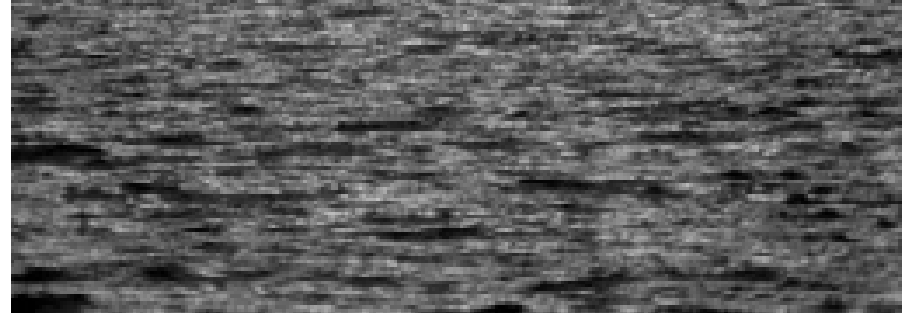

\section{Differences among African American Junior High School Students: The Effects of Skin Tone on Ethnic Identity, Self-Esteem and Cross-Cultural Behavior}

Alfiee M. Breland

Michigan State University

\section{Hardin L.K. Coleman}

University of Wisconsin-Madison

\section{Stephanie Irby Coard}

New York University Medical Center

\section{Robbie J. Steward}

Michigan State University
This article explains the results of a study conducted to assess differences among African American adolescents based on skin tone. It was hypothesized that students would demonstrate differences in self-esteem, ethnic identity and cross-cultural coping strategies based on their skin tones. 144 African American adolescents completed research packets. Results of ANOVA did not suggest any differences among the students on selfesteem, ethnic identity, or cross cultural interaction behaviors. However, significant correlations were found between self-esteem and ethnic identity and between self esteem and cross cultural interaction behaviors. Implications for counseling and education are discussed.
This research is part of a study completed to fulfill the first author's requirements for the doctoral dissertation in the Department of Counseling Psychology at the University of Wisconsin-Madison under the supervision of the second author. Alfiee M. Breland is an assistant professor in the department of Counseling, Educational Psychology and Special Education. Hardin L.K. Coleman is an associate professor in the department of Counseling Psychology, University of Wisconsin-Madison. Stephanie Irby Coard is an assistant professor of psychiatry, New York University Medical Center Department of Psychiatry, Child Study Center. Robbie J. Steward is an associate professor in the department of Counseling, Educational Psychology, and Special Education at Michigan State University. Correspondence concerning this manuscript should be addressed to Alfiee M. Breland, Department of Counseling, Educational Psychology and Special Education, Michigan State University, 437 Erickson Hall, East Lansing, Michigan 488241034. Electronic mail may be sent via Internet to breland@msu.edu.

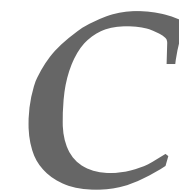

olor consciousness is a phenomenon that exists within the African American community. It is defined as a process in which African Americans "differentially attend and respond to shades of Black skin (Bond \& Cash, 1992, p. 875; Breland, 1998a; Neal \& Wilson, 1989). BoydFranklin (1991) described the pervasive nature of color consciousness within African American families and current research supports this idea. It appears that many people learn about color consciousness via verbal and non-verbal family interactions. Breland (1998a) proposed that color consciousness is an area in need of study and that researchers should attempt to determine its causes and effects. Specifically, she wrote, "Research demonstrating the relationship between skin tone bias and self-esteem... might be quite beneficial in understanding this phenomenon. Given the... discussion regarding color consciousness and African American 
racial identity, it may be important for researchers to study the relationship between the two for either correlation or causality" (p. 308). Further, Breland suggested that future research, "might also empirically document the prevalence of color consciousness among African Americans by determining if skin tone itself is a status characteristic among African Americans" (p. 308). A status characteristic is defined as any characteristic which people use to establish expectations and beliefs about other individuals (Wagner \& Berger, 1997). In other words, a status characteristic may be understood as a trait that informs how individuals treat each other. An example of a status characteristic is white skin and the associated privileges as evidenced in the writings of Peggy McIntosh (McIntosh, 1998). The following study evaluates skin tone as a status characteristic.

At present, very few studies address differential psychological, educational, and professional outcomes among African Americans. However, the limited research that exists provides strong evidence to support the notion of skin tone as a mediating variable in differential outcomes among African Americans. Hughes \& Hertel (1990) completed an analysis of one national survey of African Americans. By using data from the 1980 Survey of Black Americans (a probability household survey of 2,107 African Americans aged 18 and older), they concluded that African Americans with lighter skin have greater education, occupational prestige, personal income and family income than their darker skinned peers. Further, by controlling for variables such as age, sex, and parental socioeconomic status, they found that these differences were not due to the historical prevalence of lighter skinned African Americans in the higher socioeconomic stratum.

Keith \& Herring (1991) analyzed data from the same survey and found that skin tone has a direct effect on personal and family income, educational attainment, and occupation. Hunter (1998) and Hall (1995/96) each derived findings similar to that of the aforementioned authors. Specifically, Hunter discovered that lighter skinned African American women had, "higher educational attainment, higher personal incomes, and [were] more likely to marry higher status husbands [sic]" (p. 517). Similarly, Hall found that lighter skinned African Americans aspired to more prestigious occupations than darker skinned African Americans did. As disturbing as these trends may seem, there is little evidence offered to refute their findings. As such, it is becoming increasingly evident that skin tone may indeed be a status characteristic for African Americans.

\section{Self-Esteem, Ethnic Identity, and Cross- Cultural Interaction Behaviors}

The primary goal of this study was to determine if there are differences in the life experiences of African Americans based on skin tone. Previous research in this area has included, but not necessarily focused on, self-esteem and ethnic identity. Various researchers have hypothesized that since African Americans may receive differential treatment based on skin tone, they may internalize views that reflect such differential treatment. Breland (1998b), Coard, Breland, \& Raskin (in press), Neal \& Wilson (1989), and Okazawa-Rey, Robinson, \& Ward (1987) have all speculated, based on clinical examples and research, that there may indeed be a link between skin tone and self esteem and/or self-concept. OkazawaRey makes a very strong point about African American women that is likely applicable across genders. She wrote, "A woman's self-concept develops in part from observing and internalizing what others think about her. Consequently, the attributes society assigns to the attractive and unattractive black female have profound implications for her psychosocial development" (p.13). Similarly, researchers have speculated on the link between skin tone and ethnic identity. Hughes \& Hertel (1990) researched the effects of skin color on three aspects of "black consciousness," namely black identity, black separatism and racial self-esteem. They found, "weak but significant zero-order relationships with skin color, indicating that black identity and black separatism are more evident among those with darker skin" (p. 1110). In another study by Zweigenhaft \& Domhoff (1998), it is reported that lighter skinned African Americans are consistently more likely to occupy the higher rungs of the "power elite" or high ranking officials in business and government. With regard to the relationship between ethnic identity and skin tone, they quote a prominent African American military officer as stating that a primary reason for his acceptance among whites is that he is not "that Black" because he speaks well and is comfortable in white situations (p. 112). The authors believe that each of these examples speaks to the relationship between skin tone and self-esteem and skin tone and ethnic identity.

Little has been done to ascertain the role that skin tone plays in cross-cultural interactions. This may primarily be because little has been done to ascertain the role of skin tone on African Americans' lives in general. Further, the concept of cross-cultural interactions is a new area of research with limited empirical studies. As such, one might question the purpose of including this variable in the present study. To address that question, the authors 
present the following theory of crosscultural interactions.

Coleman, Casali, \& Wampold (2001)

hypothesize that cross -cultural interactions encompass six general categories including assimilation, acculturation, fusion, separation, integration and alternation. They suggest that any individual faced with interacting with people not from his or her primary culture may employ any of these strategies as a means of negotiating interpersonal interactions. Following is a brief description of each strategy. According to the research of LaFromboise, Coleman, \& Gerton (1993), a person who assimilates or acculturates is one who attempts to join a culture secondary to his or her primary culture. A person who separates is one who withdraws from, or avoids contact with, persons who are not members of his or her primary culture. The integration conceptualization (Berry, Kim, Power, Young, \& Bujaki, 1989) suggests that individuals can maintain their primary culture while interacting with others from different cultures. The alternation conceptualization (Ogbu \& Matute-Bianchi, 1986) suggests that it is possible for an individual to vary his or her behaviors between the primary and secondary cultures. The fusion

conceptualization assumes that individuals from different cultures, who are in constant contact with each other, will eventually merge their two cultures together to create a new culture that overrides the primary culture.

We propose that skin tone is a mediating variable in determining the number of methods of cross-cultural interaction available to African Americans and we provide the following example to support our hypothesis. Consider the aforementioned research of Zweigenhaft \& Domhoff (1998). If, as they state in their research, European Americans are more comfortable with lighter skinned African Americans because lighter skinned individuals more closely resemble European Americans, then it is possible that lighter skinned African Americans have more opportunities to interact with European Americans than their darker skinned peers (via greater access to education and higher socioeconomic status). As such, lighter skinned African Americans may have more opportunities to develop and practice various forms of cross-cultural behaviors. Therefore, we have included the examination of skin tone as a mediating variable in determining the number of methods of cross-cultural behaviors available to an African American.

In summary, the purpose of this study is to examine the relationship between skin tone and psychological variables among African Americans who differ by skin tone. It was hypothesized that there would be differences in ethnic identity, self-esteem and cross cultural interaction behaviors for the African American adolescent participants of the study.

\section{Methods}

\section{Participants}

There were 144 participants in this study. The participants were adolescents, with a mean age of 13.27 and were seventh $(49.3 \%$, $\mathrm{n}=71)$ and eighth $(50.7 \%, \mathrm{n}=73)$ grade students, in a medium sized predominantly white southeastern school system. 50.7\% (n $=73$ ) of the participants were males and 49.3\% ( $\mathrm{n}=71)$ females. Letters informing parents about the study and seeking consent for participation were sent to the participants' homes. Of the students whose parents were informed of the study, 201 offered consent. Of the 201 students who were allowed to participate in the study, 33 failed to answer the question specifying their skin tone, 2 failed to answer any of the skin tone questions and 55 were either European American or biracial (i.e., they indicated having one parent of African American descent with the other parent reported to be of a race that was not African American) and did not meet selection criteria for this study. Only the biracial and incomplete packet participants, however, were excluded from the data analysis as it seemed important to ascertain whether the "refuse to answer" participants differed significantly from the other participants. Participants completed packets in their classrooms with oversight by research assistants and their teachers. Participants were treated in accordance with the ethical guidelines for human subjects as well as the ethical guidelines for working with diverse populations.

\section{Measures}

The measures used in this study are described below.

The Multi-Construct African American Identity Questionnaire (MCAIQ) was designed to, "measure several proposed components of racial/ethnic identity in African American adolescents" (Smith and Brookins, 1997, p. 1). The test consists of 25 items used to measure socialization preferences, appearance preferences, attitudes regarding stereotypes of African Americans, and cooperative values. Participants were asked to rate each item on a scale of 1 to 5 with 5 representing strong agreement and 1 representing strong disagreement. Measures were scored by adding the numerical values of all answers to obtain scores between 25 and 125 .

According to the test developers, "higher scores on the MCAIQ are received by participants who responded to 
items endorsing in group orientations, rejecting group stereotypes; accepting positive portrayals of African Americans as a group; and espousing cooperative values" (Smith \& Brookins, 1997, p. 5). Internal reliability estimates for the measure and its subscales were found to have Cronbach's alphas ranging from .54 .87 and .56 for this sample. This measure was used to test the previously demonstrated assumption that African American adolescents differ in their ethnic identity strength based on skin tone.

The Coping with Cultural Diversity Scale (CCDS) (Coleman et al., 2001) is a 54-item scale that seeks to measure how likely an adolescent is to use different strategies for coping with cultural diversity. Although there are six proposed strategies for use in dealing with cultural diversity, this instrument incorporates only four distinct strategies including acculturation, alternation, integration, and separation. This is due to the high correlation between the fusion and integration strategies and the assimilation and acculturation strategies, which may mean that these constructs are not distinct. Currently, the scale is in the initial stages of construct validation; however a previous sample produced reliability estimates using Cronbach's alpha of .90 (Coleman et al., 1996) with the sample for the current study producing a Cronbach's alpha of .87. Further, the sample on which Coleman's data is based was $10.2 \%$ African American. This measure was used to test the assumption that skin tone might affect the number of strategies used by an African American for engaging in cross-cultural interactions.

The Rosenberg Self-Esteem Scale (RSES) (Rosenberg, 1965) is a 10-item scale used to assess self-acceptance aspects of self-esteem. Each item allows participants to provide an answer in the range of 1 to 4 with 1 representing strong disagreement and 4 representing strong agreement. "One point is scored for each item answered in the keyed direction, yielding a range from 0 (lowest self-esteem) to 40 (highest self esteem)"

(Westaway \& Wolmarans, 1992). A previous study of this measure with African Americans provided a high reliability estimate with Cronbach's alpha of .82 (Wade, 1996) and the current sample produced a Cronbach's alpha of .76. This measure was used to test the assumption that self-esteem differs for African Americans based on their skin tones.

Finally, the author administered the Breland Skin Tone and Perceptions Scale (STPT) (Breland, 1998b). This is a scale designed by the author specifically for the purpose of this study. It is a 12 item measure developed to determine participants' identification of their own skin tones and the degree to which they judge other African Americans, either positively or negatively, based on skin tone. This scale was developed because previous measures used to assess the same phenomenon presented more variables than were appropriate for this study and because no similar measures were available at the time of data collection. Specifically, with regard to the measurement of skin tone in African Americans, Bond and Cash (1992) developed a highly reliable and valid method for this purpose called the Skin Color Assessment Procedure and Skin Color Questionnaire. The measure was not included in the current study for two reasons. First, the authors made numerous attempts to secure permission to use the measure with no response from the creators and second because two of the Pantone color swatches essential to the use of the measure are no longer in production, are no longer stocked and cannot be purchased from the Pantone company.

The experimental STPT scale consists of 12 pictures of African American adolescents who vary according to skin tone. Participants are asked to select the picture of the adolescent who best fits (in their opinion) the answer to each of 12 questions. Questions 1-11 were related to aspects of social and academic ability. Question 12 on the scale was used to determine the participants' perceptions of their own skin color. The stimulus pictures were selected based on the ratings of five trained raters who rated the pictures first solely on level of attractiveness (i.e. attractive or unattractive). This was done by adjusting the skin tones of all stimulus pictures to the same medium skin tone. Only those pictures for which an inter-rater reliability of .50 was obtained were used. After raters grouped the pictures according to level of attractiveness, pictures were then altered to their original skin tone and grouped by skin tone (i.e. one category each for light, medium, and dark skin) with both genders represented. In this instance, only those pictures for which an interrater reliability of .75 was obtained were used.

The measure is scored on a continuum from a possible score of 1 to 11. Participants were given 1 point for each time they associated a dark skinned person with a negative trait or a light skinned person with a positive trait and they earned 0 points for each time they associated a light skinned person with a negative trait or a dark skinned person with a positive trait. Participants received zero points for associating medium skinned persons with any traits. Higher scores indicate general 
Table 1

Means and Standard Deviations on All Variables

\begin{tabular}{lccc}
\hline Variables & $M$ & SD & $N$ \\
\hline Ethnic Identity (MCAIQ) & 69.89 & 8.18 & 144 \\
\hline Bicultural Coping Strategy Use (CCDS) & 165.74 & 40.57 & 144 \\
\hline Skin Tone Bias (STPT) & 7.53 & 1.85 & 144 \\
\hline Self Esteem (RSES) & 26.26 & 3.09 & 144 \\
\hline
\end{tabular}

skin tone bias. As stated earlier, the measure was also used to place participants into light, medium and dark skin tone groups. Specifically, those students who selected a light skinned person as the person they most resembled were placed in the light group; those who selected a person in the middle were placed in the middle skin tone group and those who selected a dark skinned person were placed in the dark skinned group.

\section{Data Analysis}

Results

Participants were categorized as either light, medium or dark skinned according to their responses on the STPT item $12.25 \%(\mathrm{n}=36)$ considered themselves to be light

\section{Table 2}

\section{Correlation Coefficients}

\begin{tabular}{|c|c|c|c|c|c|c|}
\hline & ccdstot & meaiqto† & rsestot & selfgrp & \multicolumn{2}{|c|}{ gender } \\
\hline ccdstot & 1.0 & .15 & & $.22^{\star}$ & .03 & .03 \\
\hline mcaiqtot & & 1.00 & & $.19^{\star}$ & -.14 & .06 \\
\hline rsestot & & & & 1.00 & -.13 & .13 \\
\hline selfgrp & & & & & & \\
\hline gender & & & & & & 1.00 \\
\hline
\end{tabular}

${ }^{*}=$ significant at $\left.p<.05\right)$

KEY: (MCAIQ) ethnic identity, (CCDS) cross-cultural behavior use, (RSES) self-esteem, and (SELFGRP) self-rated skin tone, (GENDER) gender.

Skin Tone and Outcomes 7 skinned; 39.5\% ( $\mathrm{n}=57)$ considered themselves to be of medium skin tone, $12.5 \%(\mathrm{n}=18)$ considered themselves to be dark skinned and $22.9 \%(n=33)$ declined to answer this question. All other means, ranges, and standard deviations for the dependent variables are listed in table 1. The reliability estimates for each of the measures employed were as follows. For the CCDS, the reliability estimate was Cronbach's alpha of .87. Regarding the RSES, the reliability estimate was .76 (Cronbach's alpha). The reliability estimate for the MCAIQ was .56 (Cronbach's alpha). As the STPT was only used to categorize participants into skin tone groups, the alpha coefficient is not presented.

We performed a one-way analysis of variance to test the effects of skin tone on ethnic identity, self esteem and cross cultural behavior use. We found no significant differences between the skin tone groups on these variables. Table 2 presents the Pearson product correlations for the variables in the study. Positive and significant $(\mathrm{p}<.05)$ relationships were found to exist between cross cultural behavior use and self-esteem $\mathrm{r}=.22$ and ethnic identity and self-esteem $\mathrm{r}=.19$. An interpretation of the coefficients of determination $\left(\mathrm{r}^{2}\right)$, reflect a small amount of variation in ethnic identity and cross cultural behavior use by self-esteem (5\% and $4 \%$ respectively). Finally, the authors examined gender differences in the variables under study via the use of $t-$ tests. We found no differences between the genders on the variables under study.

\section{Discussion}

Regarding the main hypothesis that African Americans would differ on various psychological variables by skin tone, such was not found to be the case. This finding is in stark contrast to previous research where ethnic identity and self-esteem were found to be significantly related to skin tone. It is possible that the developmental age of the participants (13 - 15) contributed to the convergence of scores for self-esteem, which in this case was quite low. (Whaley, 1993) offered support for this assumption. In his research, he proposed that children in the stage of adolescent development are experiencing a general decrease in self-esteem as a result of the transition they experience in moving from elementary to junior high. This notion is particularly relevant for the participants of this study, half of whom were seventh graders. Regarding the skin tone independent variable and its effects on the dependent variables, it is possible that some difficulty lies in attempting to ascertain African Americans true skin tones via self-report. Because the researchers did not make judgments about the participants' skin tones, there was no means for checking the accuracy of self-ratings either via comparison or via inter-rater reliability estimates. Therefore, it is possible that the participants did not demarcate themselves appropriately into their 
true skin tone groups, which would affect outcomes on all variables studied. Considering the relatively low numbers of participants who rated themselves as dark skinned $(n=18)$, it is possible that previous findings regarding the low status attributed to dark skin might preclude an easily influenced adolescent from categorizing him or herself as such. Therefore, we would find a preponderance of participants in the medium and light categories (as was the case in this study) both of which carry either neutral or positive connotations. Finally, the literature has demonstrated that skin tone among African Americans is a very difficult subject to address (Zweigenhaft \& Domhoff, 1998) and since the skin tone portion of this study was not concealed, it is possible that scores on the measures reflect socially desirable answers and not true feelings.

As stated earlier, weak yet significant relationships were found to exist between self-esteem, ethnic identity and cross-cultural behavior use. It seems reasonable to expect that a person with higher self-esteem would have a stronger ethnic identity, given that ethnic identity is one aspect of global self-concept. Prior research in the area of ethnic identity and self-esteem has demonstrated a strong relationship between the two. Phinney (1992) and Phinney \& Alipuria (1990) found that, " self-esteem was related to ethnic identity" Phinney (1992, p.171). Further, Smith \& Brookins (1997) and Wade (1996) found a similar relationship between the two constructs and speculated that an African American with strong ethnic identity might be less likely to acknowledge the negative stereotypes put forth about African Americans, hence enhancing and confirming a high self-worth. Although the present findings are not as strong, they do seem to support this trend. Self-esteem was also related to cross cultural interactions. Coleman et al. (2001) asserts that there are between four and six strategies for use in managing cross-cultural interactions. In this study, the researchers focused on acculturation, alternation, integration, and separation for the reasons outlined regarding colinearity between some of the subscales on the CCDS measure. If we consider the fact that each of these strategies requires some skill to be successful, then it seems reasonable to expect that a person with higher self-esteem would feel more competent and skilled in using more of the strategies. Further, based on the aforementioned literature, it is possible to speculate that a person who holds him or herself in high regard may be more likely to attract others to him or her than someone with lower self-regard. Note Whaley's (1993) culture specific reference to the work of Susan Harter. He suggests that for African American children, self-esteem is related to the child's perception of his or her level of competence in areas that are important to him or her. As such, it is probable that the child who feels more competent in engaging in cross-cultural behaviors would derive some sense of his or her higher self-esteem from being able to use different strategies. Conversely, an adolescent with more trouble in and fewer opportunities for practicing the use of the cross-cultural interaction skills might have lower self-esteem.

\section{Conclusions and Limitations}

The results of this study regarding skin tone might lead one to believe that the prior literature focused on examining intra-group differences among African Americans is no longer necessary or relevant. Although the findings of this study did not corroborate previous findings, this area is still one in which further research should be conducted. It is possible that the limitations of this study, including the small sample size and the large number of participants who elected not to categorize themselves based on their skin tones, may have contributed to the lack of support for the main hypothesis. It is further possible that use of the STPT may be partially to blame for the lack of corroboration for the main hypothesis. The novelty of the measure, coupled with the fact that it was developed specifically for use in this study is a consideration. The stimulus objects in the pictures were not dressed in a uniform manner and therefore may have influenced participants via style of dress. In addition, there may be differences in perceptions of skin tone and level of attractiveness between the adults (who acted as raters to select the pictures) and adolescents (who served as participants). Future research might address the manners in which data on the categorization of African Americans by skin tone is collected. It is important that future work in this area adapt measures for inclusion to provide a more reliable and well-tested means of measuring what can often be a very subjectively observed trait.

We suggest the following three conclusions drawn from the findings of this study. School and community counselors and psychologists who work with African American adolescents should note the relationship between self-esteem and ethnic identity. It is possible that increases in African American adolescents' ethnic pride will affect increases in their self-esteem. Further, given the relationship between self-esteem and cross cultural interactions, it seems important for those counselors and psychologists working in highly culturally diverse environments to help students develop skill in utilizing multiple methods of cross cultural interaction so that they 
might derive higher self-esteem. The results from this study suggest that aspects of ethnic identity and crosscultural interaction interacting with self-esteem seem quite important to the psychosocial development of African American adolescents.

\section{References}

Berry, J. W., Kim, U., Power, S., Young, M., \& et al. (1989). Acculturation attitudes in plural societies. Applied Psychology: An International Review, 38(2), 185-206.

Bond, S., \& Cash, T. F. (1992). Black beauty: Skin color and body images among African-American college women. Journal of Applied Social Psychology, 22(11), 874-888.

Boyd-Franklin, N. (1991). Recurrent themes in the treatment of AfricanAmerican women in group psychotherapy. Women $\mathcal{E}$ Therapy, 11(2), 25-40.

Breland, A. M. (1998a). A model for differential perceptions of competence based on skin tone among African Americans. Journal of Multicultural Counseling \& Development, 26(4), 294-311.

Breland, A. M. (1998b). Airing dirty laundry: Reasons and processes by which skin tone stratification continues to be a pervasive aspect of the African-American community. Dissertation Abstracts International: Section B: The Sciences \& Engineering, 59(1-B), 0457.

Coard, S., Breland, A. M., \& Raskin, P. (in press). Perceptions of and preferences for skin color, Black racial identity, and self-esteem among African Americans. Journal of Applied Social Psychology.

Coleman, H. L. K., Casali, S. B., \& Wampold, B. E. (2001). Adolescent strategies for coping with cultural diversity. Journal of Counseling $\mathcal{E}$ Development Special Issue, 79(3), 356-364.

Hall, R. E. (1995/96). Dark skin and the cultural ideal of masculinity. Journal of African American Men, 1(3), 37-62.

Hughes, M., \& Hertel, B. R. (1990). The significance of color remains: A study of life chances, mate selection, and ethnic consciousness among Black Americans. Social Forces, 68(4), 1105-1120.

Hunter, M. L. (1998). Colorstruck: Skin color stratification in the lives of African American women. Sociological Inquiry, 68(4), 517- 535.

Keith, V. M., \& Herring, C. (1991). Skin tone and stratification in the Black community. American Journal of Sociology, 97(3), 760-778.

LaFromboise, T., Coleman, H. L., \& Gerton, J. (1993). Psychological impact of biculturalism: Evidence and theory. Psychological Bulletin, 114(3), 395-412.
McIntosh, P. (1998). White privilege: Unpacking the invisible knapsack. In M. McGoldrick (Ed.), Re-visioning family therapy: Race, culture, and gender in clinical practice (pp. 147-152). New York: The Guilford Press.

Neal, A. M., \& Wilson, M. L. (1989). The role of skin color and features in the Black community: Implications for Black women and therapy. Clinical Psychology Review, 9(3), 323-333.

Ogbu, J. U., \& Matute-Bianchi, M. A. (1986). Understanding Sociocultural Factors: Knowledge, Identity and Social Adjustment. In Language Minority Children/Bilingual Education Office (Ed.), Beyond Language: Social and cultural factors in schooling (pp. 73-142). Los Angeles: California State Department of Education Evaluation, Dissemination and Assessment Center, California State University.

Okazawa-Rey, M., Robinson, T., \& Ward, J. V. (1987). Black women and the politics of skin color and hair. Women $\mathcal{E}$ Therapy Special Issue: Women, power, and therapy: Issues for women, 6(1-2), 89-102.

Phinney, J. S. (1992). The multigroup ethnic identity measure: A new scale for use with diverse groups. Journal of Adolescent Research, 7(2), 156-176.

Phinney, J. S., \& Alipuria, L. L. (1990). Ethnic identity in college students from four ethnic groups. Journal of Adolescence, 13(2), 171-183.

Rosenberg, M. (1965). Society and adolescent self-image. Princeton: Princeton University Press.

Smith, E. P., \& Brookins, C. C. (1997, Nov). Toward the development of an ethnic identity measure for African American youth. Retrieved September 16, 1999, from http: / / www.proquest.umi.com/pdqweb

Wade, T. J. (1996). The relationships between skin color and selfperceived global, physical, and sexual attractiveness, and selfesteem for African Americans. Journal of Black Psychology, 22(3), 358373.

Wagner, D. G., \& Berger, J. (1997). Gender and interpersonal task behaviors: Status expectation accounts. Sociological Perspectives, 40(1), 1-32.

Westaway, M. S., \& Wolmarans, L. (1992). Depression and self-esteem: Rapid screening for depression in Black, low literacy, hospitalized tuberculosis patients. Social Science \& Medicine, 35(10), 1311-1315.

Whaley, A. L. (1993). Self-esteem, cultural identity, and psychosocial adjustment in African American children. Journal of Black Psychology Special Issue: Emotional development of African American children, 19(4), 406-422.

Zweigenhaft, R. L., \& Domhoff, G. W. (1998). Diversity in the power elite: Have women and minorities reached the top? New Haven, CT: Yale University Press. 